\title{
Detection of homoeologous chiasma formation in Triticum durum $x$ Thinopyrum bessarabicum hybrids using genomic in situ hybridization
}

\author{
I. P. KING, K. A. PURDIE, S. E. ORFORD, S. M. READER \& T. E. MILLER \\ Cambridge Laboratory, JI Centre, Colney Lane, Norwich NR4 7UJ, U.K.
}

\begin{abstract}
Genomic in situ hybridization (GISH) was used to study the nature of homoeologous chiasma formation in crosses between Triticum durum cv. Creso, homozygous for the phlc mutation and Thinopyrum bessarabicum. The relative frequencies of wheat/wheat and wheat/Th. bessarabicum chiasma formation were determined. Pairing between apparently non-homologous $T h$. bessarabicum chromosomes was also observed. The potential of GISH as a tool for analysing homoeologous chiasma formation in wheat/alien hybrids is discussed.
\end{abstract}

Keywords: genomic in situ hybridization, homoeologous recombination, Thinopyrum bessarabicum, Triticum durum.

\section{Introduction}

Wheat, which belongs to the tribe Triticeae, can be sexually hybridized with many of its wild relatives. Although the genomes of wheat and the alien species in such hybrids are related, with a few notable exceptions such as wheat $x$ Aegilops speltoides, very little chiasma formation between wheat/wheat and wheat/alien homoeologous chromosomes occurs (Riley et al., 1959). This is the result of the action of pairing control genes, located on wheat chromosomes, which restrict chiasma formation to homologous chromosomes (for review see Gale \& Miller, 1987). The strongest of the pairing control genes, $P h 1$, is located on the long arm of wheat chromosome 5B (Okamoto, 1957; Riley \& Chapman, 1958). In hybrids which lack Phl, obtained by crossing alien species with lines of wheat which either lack chromosome 5B or in which $P h 1$ has been mutated (Sears, 1977; Giorgi, 1983), homoeologous pairing can occur.

The study of the relative frequency of homoeologou's chiasma formation between wheat chromosomes and alien chromosomes in high pairing hybrids is important for two reasons. First, alien species have been found to carry agronomically useful genes. The introduction of these genes to wheat can be achieved

Correspondence: I. P. King. by recurrently backcrossing high pairing wheat $x$ alien hybrids with the wheat parent and selecting for the presence of the target gene at each generation. The ultimate aim of this process is to introduce into wheat a small segment of alien chromatin containing the target gene (for review see Gale \& Miller, 1987). A knowledge of the relative frequency of wheat/alien chiasma formation in wheat/alien hybrids is of interest as it gives an indication of the likelihood of transferring alien genes to wheat. Secondly, the relative frequency of wheat/alien and wheat/wheat homoeologous chiasma formation in high pairing wheat $x$ alien hybrids is important as it provides a measure of the closeness of the relationship between the genomes of wheat and the genome(s) of the wild species.

The determination of the frequency of homoeologous chiasma formation in wheat $x$ alien hybrids requires that the chromosomes of each parent can be identified in pollen mother cells (PMCs). One way in which this can be determined is to highlight differentially either the wheat or the alien chromosomes using genomic in situ hybridization (GISH) (Schwarzacher et al., 1992). The work in this paper describes the use of GISH to determine the nature and frequency of homoeologous chiasma formation in a hybrid between the macaroni wheat Triticum durum cv. Creso, homozygous for the phlc mutation, and the sand couch grass Thinopyrum bessarabicum which has been shown to 
carry a gene(s) conferring salt tolerance (Forster et al., 1987, 1988).

\section{Materials and methods}

The plant material used consisted of the $T$. durum Desf. cv. Creso homozygous for the phIc mutation $(2 n=4 x=28, \mathrm{AABB})$ and Th. bessarabicum (Savul. and Rayss) Löve ( $\left.2 n=2 x=14, E^{\text {b' }} E^{\text {b }}\right)$.

Creso phlc $\times$ Th. bessarabicum hybrids were produced as follows: emasculated spikes of Creso phlc were pollinated with $T h$. bessarabicum; the following day the internodes and the florets of the pollinated spikes were treated with $10 \mathrm{mg} \mathrm{l}^{-1}$ 2,4-dichlorophenoxyacetic acid (2,4-D) as described by Laurie \& Reymondie (1991) with the modification that $10 \mathrm{mg} \mathrm{l}^{-1}$ of $\mathrm{AgNO}_{3}$ was added to the 2,4-D solution (Laurie \& O'Donoughue, 1993). Embryo rescue was performed as described by Laurie \& Reymondie (1991) with the exception that Gamborg B5 medium was used instead of Difco Orchid agar.

DNA was extracted from leaves of Creso phlc and Th. bessarabicum by the method described by Sharp et al. (1988). Probe preparation and in situ hybridization were performed as described by Schwarzacher et al. (1992) with the following modifications. Both Th. bessarabicum, used as probe, and Creso phlc, used as unlabelled blocking DNA, were mechanically sheared using a Decon Fs minor sonicating water bath. Creso phlc was used as unlabelled blocking DNA at concentrations between 60 and 80 times greater than that of the Th. bessarabicum probe DNA (70 ng per slide). Anthers from Creso phlc $\times$ Th. bessarabicum hybrids containing PMCs at metaphase I of meiosis were placed in 45 per cent acetic acid for 10 min prior to slide preparation. Slides were analysed by epifluorescence microscopy using a Nikon Microphot-SA microscope. Photographs were taken using Kodak Ekta 1000 ASA print film.

\section{Results}

Three Creso phlc $\times$ Th. bessarabicum hybrid embryos were obtained from six pollinated spikes. Each of the three embryos rescued gave rise to a mature 21chromosome plant. The majority of PMCs examined contained dense cytoplasm. It was not possible to score these PMCs for the frequency of wheat/wheat and wheat/Th. bessarabicum chromosome pairing as both the wheat and $T h$. bessarabicum chromosomes appeared to fluoresce with equal intensity. However, in 66 PMCs, which appeared to contain considerably less cytoplasm, the wheat and Th. bessarabicum chromosomes could be clearly distinguished.

In total, 79 chiasmata resulting in the formation of 75 chromosome associations ( 74 bivalents and one trivalent), were observed (Table 1). In no case were paired chromosome arms with more than one chiasma observed. Rod bivalents were by far the most frequent type of configuration observed, (94.6 per cent). Only three ring bivalents ( 4 per cent) and a single trivalent (1.3 per cent) were seen. Of the 79 chiasma scored 65 (82.3 per cent) occurred between wheat chromosomes presumably A and B genome homoeologues (Fig. 1a), 10 (12.7 per cent) between wheat chromosomes and

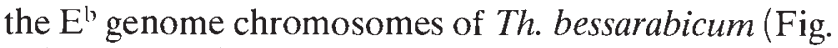
$1 \mathrm{~b})$ and four (5.1 per cent) were between $\mathrm{E}^{\mathrm{b}}$ genome chromosomes (Fig. 1c). These latter four must be the result of non-homologous Th. bessarabicum/Th. bessarabicum chromosome pairing. The number of wheat/wheat bivalents in the 66 PMCs examined ranged from 0 to 3 per PMC as compared with a range of 0 to 1 for the wheat/Th. bessarabicum and the Th. bessarabicum/Th. bessarabicum bivalents.

Overall, chiasma formation between a wheat and $T h$. bessarabicum chromosome was observed in nine out of 66 (13.6 per cent) of the PMCs examined.

Table 1 The number of wheat/wheat, wheat/Th. bessarabicum and Th. bessarabicum/Th. bessarabicum homoeologous chromosome pairing configurations in 66 PMCs from three Creso phlc $\times$ Th. bessarabicum hybrids

\begin{tabular}{|c|c|c|c|c|c|c|}
\hline \multicolumn{2}{|l|}{$\mathrm{W} / \mathrm{W}$} & \multicolumn{2}{|l|}{$\mathrm{W} / T h$} & \multirow{3}{*}{$\begin{array}{l}\mathrm{W} / \mathrm{W} / T h \\
\text { Trivalent }\end{array}$} & \multicolumn{2}{|l|}{$T h / T h$} \\
\hline Rod & Ring & Rod & Ring & & Rod & Ring \\
\hline Bivalent & Bivalent & Bivalent & Bivalent & & Bivalent & Bivalent \\
\hline 60 & 2 & 7 & 1 & 1 & 4 & 0 \\
\hline
\end{tabular}

$\mathrm{W}=$ wheat; $T h=T h$. bessarabicum. 
Fig. 1 PMCs showing (a) two wheat bivalents (open triangles), (b) a wheat/ Th. bessarabicum bivalent (closed triangle) and a wheat/wheat bivalent (open triangles) and (c) a single $T h$. bessarabicum/Th. bessarabicum bivalent (closed triangles).
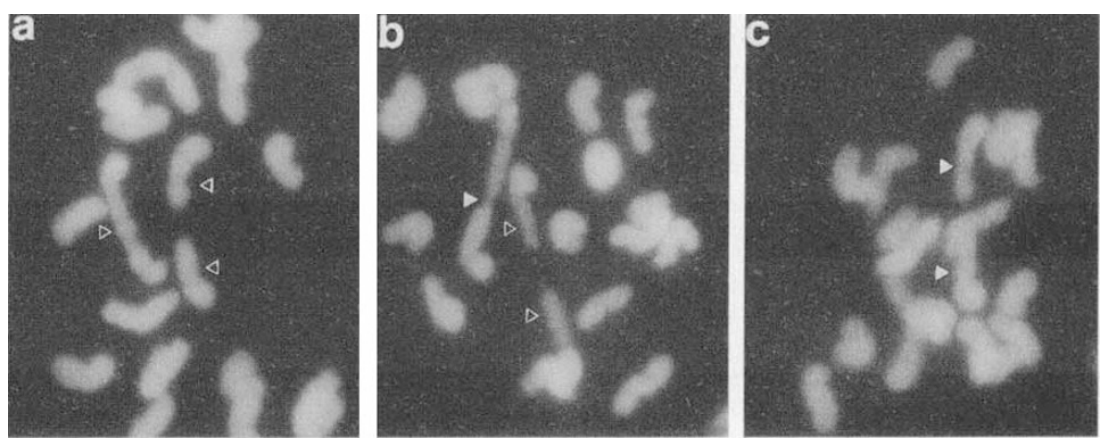

\section{Discussion}

Using GISH it was possible to determine the relative frequency of wheat/wheat ( 82.3 per cent), wheat/Th. bessarabicum (12.7 per cent) and Th. bessarabicum/Th. bessarabicum (5.1 per cent) recombination. The frequency of wheat/wheat homoeologous pairing was considerably higher than wheat/Th. bessarabicum homoeologous pairing, demonstrating that the $\mathrm{A}$ and $\mathrm{B}$ genomes of wheat are more closely related to each other than to the $\mathrm{E}^{\mathrm{b}}$ genome of Th. bessarabicum. A study of the relative frequencies of wheat/wheat and wheat/alien recombination provides a means by which genomic relationships can be analysed. It is expected that an alien species more closely related to wheat than Th. bessarabicum would have a higher frequency of wheat/alien recombination while a more distantly related species would have a lower frequency of recombination. However, such information should be treated with caution because if a wild species carries extensive translocation differences relative to wheat the frequency of wheat/alien recombination may be significantly reduced. Moreover, alien species may also carry genes which promote or restrict chromosome pairing.

Chiasmata have been demonstrated to be the result of exchange events between chromatids of different chromosomes (Tease \& Jones, 1978). Thus the study of chiasma formation provides a valuable method of estimating the frequency of recombination in hybrids (Naranjo et al., 1989). The frequency of chiasma formation between wheat and alien chromosomes is of interest as it gives an indication of the ease or the difficulty with which a chromatin segment from a wild species can be introgressed into wheat. Th. bessarabicum, the wild species used in this study, is of interest as it carries genes conferring salt tolerance (Forster et al., 1987, 1988). Soils that contain a high concentration of salt are proving to be a major world problem which is increasing due to the extensive use of irrigation. In this work wheat/Th. bessarabicum chiasmata were shown to occur in 13.6 per cent of the PMCs examine, indicat- ing that it should be possible to transfer a gene conferring salt tolerance from $T h$. bessarabicum to wheat.

Four Th. bessarabicum/Th. bessarabicum rod bivalents were observed in four different PMCs. The rationale for pairing between apparently unrelated chromosomes is unknown. However, similar pairing has previously been observed between non-homologous rye chromosomes in wheat $x$ rye hybrids (Hutchinson et al., 1983). One possibility is that in the absence of a pairing partner chiasma formation occasionally occurs between homologous repetitive DNA sequences which are distributed throughout the genome resulting in bivalent formation between otherwise unrelated chromosomes.

It would not have been possible to determine the frequency of homoeologous chiasma formation nor the presence of Th. bessarabicum/Th. bessarabicum bivalents using Feulgen or carmine stained preparations since these techniques would not distinguish wheat from Th. bessarabicum chromosomes. Although multivalents, which have previously been assumed to indicate wheat/alien chiasma formation, could have been identified using these techniques, in this work considerably more wheat/Th. bessarabicum bivalents (eight) were observed than wheat/Th. bessarabicum multivalents (one). This indicates that the frequency of multivalent formation cannot be used as a reliable estimate of the relative frequency of wheat/Th. bessarabicum chiasma formation.

To date the only other method of accurately studying homoeologous pairing in wheat/alien $F_{1}$ hybrids is by using C-banding (Hutchinson et al., 1983; Jouve \& Giorgi, 1986). However, to determine the frequencies of homoeologous chiasma formation accurately it is necessary that each of the alien chromosomes has a pattern distinct from those of the wheat chromosomes which may not always be the case. This is not a problem using GISH where alien chromosomes can be clearly distinguished providing that the wild species is sufficiently diverged from wheat.

In this work total genomic Th. bessarabicum DNA was used as a probe allowing the $\mathrm{E}^{\mathrm{b}}$ genome chromo- 
somes to be distinguished from the A and B genomes of wheat and hence it was possible to determine the relative frequency of wheat/wheat and wheat/Th. bessarabicum chiasma formation. However, it was not possible to determine if the frequency of pairing of the $\mathrm{A}$ and $\mathrm{B}$ genome chromosomes with the $\mathrm{E}^{\mathrm{b}}$ genome chromosomes was the same. A difference in the frequency of chiasma formation would indicate that one of the wheat genomes might be more closely related to the $\mathrm{E}^{\mathrm{b}}$ genome than the other. It is therefore desirable that techniques are developed such that all the chromosomes of each genome in a hybrid can be identified. In the case of the T. durum $\times$ Th. bessarabicum hybrids, used in this work, this might be achieved by using two total genomic probes, i.e. Th. bessarabicum and $T$. urartu (A-genome), labelled with different fluorochromes, and unlabelled blocking DNA from a third genome, i.e. Ae. speltoides which is thought to be closely related to the $\mathrm{B}$ genome donor.

One drawback with using GISH to study the frequency and nature of wheat/alien chiasma formation in the Creso $\times$ Th. bessarabicum hybrids was that the frequency of scorable PMCs was low. This appeared to be related to the variable amount of cytoplasm retained by the PMCs in the preparations, i.e. the in situ technique failed to work in cells with dense cytoplasm but worked in cells which appeared to contain less cytoplasm. Recent work (manuscript in preparation), however, has revealed that the frequency of scorable PMCs can be considerably increased by treating slides with proteinase $\mathrm{K}$ prior to the addition of the probe mix.

In our hands GISH proved to be a robust and valuable technique for studying meiosis in a wheat $x$ alien hybrid.

\section{Acknowledgement}

I. P. King acknowledges the Overseas Development Administration for financial support.

\section{References}

FORSTER, B. F., GORHAM, J. AND MILLER, T. E. 1987. Salt tolerance of an amphidiploid between Triticum aestivum and Agropyron junceum. Plant Breeding, 98, 1-8.

FORSTER, B. F., MILLER, T. E. AND LAW. C. N. 1988. Salt tolerance of two wheat-Agropyron junceum disomic addition lines. Genome, 30, 559-564.

GALE, M. D. AND MILLER, T. E. 1987. The introduction of alien genetic variation in wheat. In: F.G.H. Lupton (ed.) Wheat Breeding: its scientific basis, pp. 173-210.

GIORGI, B. 1983. Origin, behaviour and utilization of a Phl mutant of durum wheat, Triticum turgidum (L.) var. durum. In: Sakamoto, S. (ed.) Proc. 6th Int. Wheat Genet. Symp. Kyoto, Japan, pp. 1033-1040.

HUTCHINSON, J., MILLER, T. E. AND READER, S. M. 1983. C-banding at meiosis as a means of assessing chromosome affinities in the triticeae. Can. J. Genet. Cytol., 25, 319-323.

JOUVE, N. AND GIORGI, B. 1986. Analysis of induced homoeologous pairing in hybrids between $6 \mathrm{x}$ triticale phl mutant and Triticum aestivum L. Can. J. Genet. Cytol., 28, 696-700.

LAURIE, D. A. AND REYMONDIE, S. 1991. High frequencies of fertilization and haploid seedling production in crosses between commercial hexaploid wheat varieties and maize. Plant Breeding, 106, 182-189.

LAURIE, D. A. AND O'DonoughuE, L. S. 1993. Wheat $x$ maize crosses for the production of wheat haploids. Agriculture and Forestry, 25, in press.

NARANJO, T., FERNANDEZ-RUEDA, P., GOICOECHEA, P. G., ROCA, A. AND GIRALDEZ, R. 1989. Homoeologous pairing and recombination between the long arms of group 1 chromosomes in wheat x rye hybrids. Genome, 32, 293-301.

окамото, м. 1957. Asynaptic effect of chromosome V. Wheat Inf. Serv., 5, 6.

RILEY, R. AND CHAPMAN, V. 1958. Genetic control of the cytologically diploid behaviour of hexaploid wheat, Nature, 182, 713-715.

RILEY, R., CHAPMAN, V. AND KIMBER, G. 1959. Genetic control of chromosome pairing in intergeneric hybrids with wheat. Nature, 183, 1244-1246.

SCHWARZACHER, T., ANAMTHAWAT-JONSSON, K., HARRISON, G. E., ISLAM, A. K. M. R., JIA, J. Z., KING, I. P., LEITCH, A. R., MILLER, T. E., READER, S. M., ROGERS, w. J., SHI, M. AND HESLOP-HARRISON, J. S. 1992. Genomic in situ hybridization to identify alien chromosome segments in wheat. Theor. Appl. Genet., 84, 778-786.

SEARS, E. R. 1977. An induced mutant with homoeologous pairing in common wheat. Can. J. Genet. Cytol., 19, 585-593.

SHARP, P. J., KRIES, M., SHEWRY, P. R. AND GALE, M. D. 1988. Location of $\beta$-amylase sequences in wheat and its relatives. Theor. Appl. Genet., 75, 286-290.

TEASE, C. AND JONES, G. H. 1978. Analysis of exchanges in differentially stained meiotic chromosomes of Locusta migratoria after BrdU-substitution and FPG staining. 1. Crossover exchanges in monochiasmate bivalents. Chromosoma, 69, 163-178. 